\title{
Quality of Life in Pediatric Patients with Minimal Change Disease
}

\author{
Mohamed Abdelaziz El-Gamasy ${ }^{1^{*}}$ and Mohamed Mahmoud Abdelmageed ${ }^{2}$ \\ ${ }^{1}$ Assistant Professor of Pediatrics, Faculty of Medicine, Tanta University, Egypt \\ ${ }^{2}$ Pediatric Department of Tanta Faculty of Medicine, Tanta University, Egypt
}

Received: November 6, 2017; Accepted: November 10, 2017; Published: November 13, 2017

*Corresponding author: Mohamed Abdelaziz El-Gamasy, Assistant Professor of Pediatrics, Faculty of Medicine, Tanta University, Egypt, E-mail: mgamsy@gmail.com

\begin{abstract}
Objectives: This study was conducted to assess the quality of life (QOL) in children between 2 and 18 years of age with Minimal Change Disease (MCD) as the commonest variant of primary idiopathic nephrotic syndrome (NS) using validated arabic version of Pediatric Quality of Life Inventory (PedsQL ${ }^{\mathrm{TM}} 4.0$ Generic Core Scales).

Subjects: This cross-sectional comparative study was conducted in Tanta University Hospital (TUH), Pediatric nephrology inpatient unit or outpatient clinic between October 2016 and October 2017. In this questionnaire-based study, 100 children with primary idiopathic NS and an equal number of age-matched controls with other chronic diseases were included. Their clinical and demographic details were recorded and QOL was assessed using PedsQL ${ }^{\text {TM }} 4.0$ Generic Core Scales.

Conclusions: The present study shows that the overall QOL in children with MCD was better than in children with other chronic illnesses.
\end{abstract}

Keywords: Quality of Life; Pediatric; Minimal change disease;

\section{Introduction}

Nephrotic syndrome (NS) in children is a common, yet challenging, relapsing, and remitting renal disorder and exhibits a heterogeneous clinical phenotype ranging from a single episode, infrequently relapsing, frequently relapsing to steroid-resistant disease [1]. Although $80 \%$ of these children are corticosteroid responsive, nearly half of them demonstrate a frequently relapsing or steroid-dependent course, often resulting in multiple complications, hospitalizations, or even chronic renal failure [2]. Not infrequently, NS spans a significant portion of a child's formative years. Characteristics inherent to NS including edema, relapsing nature of the disease, and repeated exposure to steroids or other immunosuppressive drugs pose a challenge to quality of life (QOL) in these children [3]. Hence, a formal evaluation of the impact of the disease on physical, emotional, social, and school performance and health-related QOL (HRQOL) is imperative to provide comprehensive and holistic patient care. Considering the paucity of information on QOL in these patients in general and Arab children in particular, we conducted the present study $[2,3]$.

The aim of this work was to evaluate the HRQOL in children with MCD and delineate the HRQOL domains in various clinical subtypes of INS. The primary objective was to assess the QOL in children between 2 and 18 years of age with the commonest idiopathic NS (MCD) using Pediatric Quality of Life Inventory (PedsQL $^{\mathrm{TM}}$ 4.0 Generic Core scales) in relation to controls. The secondary objective was to evaluate the physical, emotional, and social domains affected in various clinical phenotypes of pediatric MCD.

\section{Patients and Methods}

\section{Design and Setting}

This cross-sectional, comparative, questionnaire-based study was conducted in Tanta university pediatric nephrology inpatient unit and outpatient clinic between October 2016 and October 2017 after approval from the research ethical committee of the Faculty of Medicine of Tanta University and informed written or verbal consents from parents of included patients.

\section{Sample size \& sampling}

The cases included children with MCD, attending the pediatric nephrology Unit and or outpatient clinic of Pediatric Department of TUH. 200 children were selected then they were randomly subdivided into 2 groups, group 1 included 100 known cases of MCD aged 2-18 years and group 2 included 100 age matched children attending the general pediatric outpatient clinic and other pediatric subspecialty clinics.

\section{Inclusion criteria}

All children of both sexes with INS who fulfilled clinical and or histopathological criteria of MCD. NS was defined by the International Study for Kidney Diseases in Children (ISKDC) criteria which included heavy proteinuria, hypoalbuminemia (serum albumin $<2.5 \mathrm{~g} / \mathrm{dL}$ ), hyperlipidemia (serum cholesterol $>200 \mathrm{mg} / \mathrm{dL}$ ), and edema [4]. Nephrotic range proteinuria was defined as early morning urine protein $3+/ 4+$ (on dipstick) and spot protein: creatinine ratio $>2 \mathrm{mg} / \mathrm{mg}$. INS was pheotypically classified according to Bagga and Strivastava [5]. The cases group was managed by the routine protocol of management of nephrotic syndrome [5]. 


\section{Exclusion criteria}

Children less than 2 years of age, as they mostly could not MCD or NS secondary to systemic diseases. The controls were required to have not any clinical or biochemical features of INS

\section{Methods}

All patients were subjected to:

\section{Full History Taking}

All the parents of included subjects were explained about the purpose of the study, and confidentiality of data collected was ensured .History included personal data (name, age, sex, address, telephone,) history of diseases or developmental disorders, socioeconomic level, age of 1st presentation, duration of disease, frequency of relapses was measured in studied cases group.

Subtype of steroid sensitive nephrotic syndromes which were:

a. Frequent relapsing nephrotic syndrome: which was defined as relapse $\geq 4$ times per year or $\geq 2$ times per 6 months. Relapse was defined as recurrence of proteinuria [urine albumin dipstick $\geq 2+$ on 3 consecutive days, most often in association with recurrence of edema

b. Infrequent relapsing nephrotic syndrome: which was defined as relapse $<4$ times per year or $<2$ times per 6 months.

c. Steroid dependent nephrotic syndrome :which was defined as relapse every time with the withdrawal of steroids from every day to every other day therapy [5].

\section{Thorough Clinical Examination}

Included anthropometric measurements and vital signs especially arterial blood pressure which was measured by auscultatory method using a mercury sphygmomanometer, in the semisetting position after 10 minutes of rest, using an appropriate sized cuff and was taken as the mean value of 3 successive readings in 3 different days, presence of edema or presence of any complication either of the disease or for immunosupreesant agents used for treatment (e.g.prednisolone, Cyclosporin A, Cyclophosphamide, Mycophenolate moefetyl or others)

\section{Routine Laboratory Investigations(in the 1st visit only) Including}

Boiling test for urine sample, complete urine analysis, 24 hr. urinary proteins, complete blood count (CBC), erythrocyte sedimentation rate(ESR), serum albumin, total serum cholesterol and kidney function tests (BUN, serum creatinne).

\section{QOL Assessment}

Validity and reliability of used questionnaire- Data were collected using a validated Arabic version of structured questionnaire of PedsQL ${ }^{\mathrm{TM}} 4.0$ Generic Core Scale.The instrument was translated by professional translators from English to the regional language (Arabic Language) and retranslated to English to compare with the original version. It took approximately
30 min to gather the required information from the parents of included subjects. The tool was piloted on 20 parents before initiation of the study.

The tool evaluates the QOL in five domains: physical functioning (Eight Items), psychosocial functioning including emotional functioning (five items), social functioning (five items), and school functioning ( 5 items). The PedsQL scores range from 0 to 100 points. Recall time was one month and a 5-point response scale was used from 0 (never a problem) to 4 (almost always a problem). The scores of each item were then reversed and linearly transformed into a $0-100$ scale $(0=100,1=75,2=50$, $3=25$, and $4=0$ ), with higher PedsQL scores indicating a better QOL. Parameters studied were QOL total score, QOL in social, emotional, physical, and school domains [4].

The questionnaires were filled through an interview with the child and the Caregiver, the questions were asked in lay language to the child if he was older than 6 years and to the caregiver if the child was $\leq 6$ years [4].

\section{Sample Size and Statistical Analysis}

A convenient sample size of 200 patients was chosen based on the duration of the study. A total of 100 cases and an equal number of controls were recruited. Continuous variables with normal distribution were expressed as mean values and standard deviation and compared using Student's t-test, whereas those not normally distributed were expressed as median and range and analyzed using Mann-Whitney U-test. Categorical variables were compared using Chi-square test or Fisher's exact test.

One-way analyses of variance or Kruskal - Wallis tests were used to analyze statistical differences between three or more groups for parametric and nonparametric data, respectively. $\mathrm{P}<$ 0.05 was considered statistically significant. Data were evaluated using Statistical Package for the Social Science (SPSS) software version 16.0 (SPSS Inc., Chicago, IL, USA). Predictive risk factors for poor total and school performance scores were determined by univariate analysis using Student's t-test [6].

\section{Results}

Total of one hundred studied nephrotic patients with MCD and an equal number of controls with other chronic diseases aged 2.2-15 year and 3.5-13 years, respectively, were included in the study. Table 1 illustrated the demographic details of the studied cases and controls. Among children with MCD, 32\% had first attack or infrequently relapsing variant while $68 \%$ had difficult to treat clinical phenotypes (frequently relapsing, steroiddependent, and steroid-resistant varieties). The median duration of the disease was 33 months. The disease profile among the control children included chronic diseases, idiopathic epilepsy (30 cases), persistant asthma (15 cases), hemophilia (6 cases), beta thalassemia major (4 cases), hyperthyroidism (8 cases), hypothyroidism (14 cases), congenital heart disease (3 cases), chronic rheumatic heart disease (8 cases), attention deficit hyperactivity disorder (4 cases), autoimmune hemolytic anemia (4 cases), congenital dyserythro-poietic anemia (2 cases), and hypereosinophilic syndrome (2 case). Comparison of baseline 
characteristics of cases and controls revealed no statistical difference $(p>0.05)$. Table 2 compared between the cases and controls according to the median PedsQL ${ }^{\mathrm{TM}} 4.0$ Generic Core Scale scores. It were found that the median PedsQL ${ }^{\text {TM }} 4.0$ Generic Core Scale scores were higher in cases when compared to controls. There were significantly better scores in physical $(P=0.004)$, emotional $(\mathrm{P}=0.03)$, and social functioning $(\mathrm{P}=0.01)$ among the former. However, the school performance scores in cases versus controls were not significantly different from each other $(\mathrm{p}=0.75)$. (Table 3) summarized comparison between QOL in different clinical phenotypes of the studied MCD and concloded no statistically significant differences between different clinical phenotypes regarding QOL ( $p>0.05$ ). Univariate analysis for poor total QOL scores was performed for this study. Poor scores were defined as scores below the $25^{\text {th }}$ percentile of the median total QOL score of cases. Demographic details such as age [OR: 0.5 (95\% CI: 0.1-2.3)], gender [OR: 0.38(95\% CI: 0.07-2.1)], duration of illness [OR: 0.86 (95\% CI: 0.2-4)], steroid resistance [OR: 3.3 (95\% CI: 0.36-30.1)], complications related to therapy [OR: 1.8 (95\% CI: 0.2-17.6)], and per capita income and history of peritonitis [OR: 1.4 (95\% CI: 0.14-14.1)] did not significantly influence the total QOL scores among our studied nephrotic children ( $\mathrm{P}>0.05)$. Similarly, univariate analysis for predictors of poor school performance QOL scores did not reveal the a fore mentioned variables to be significant.

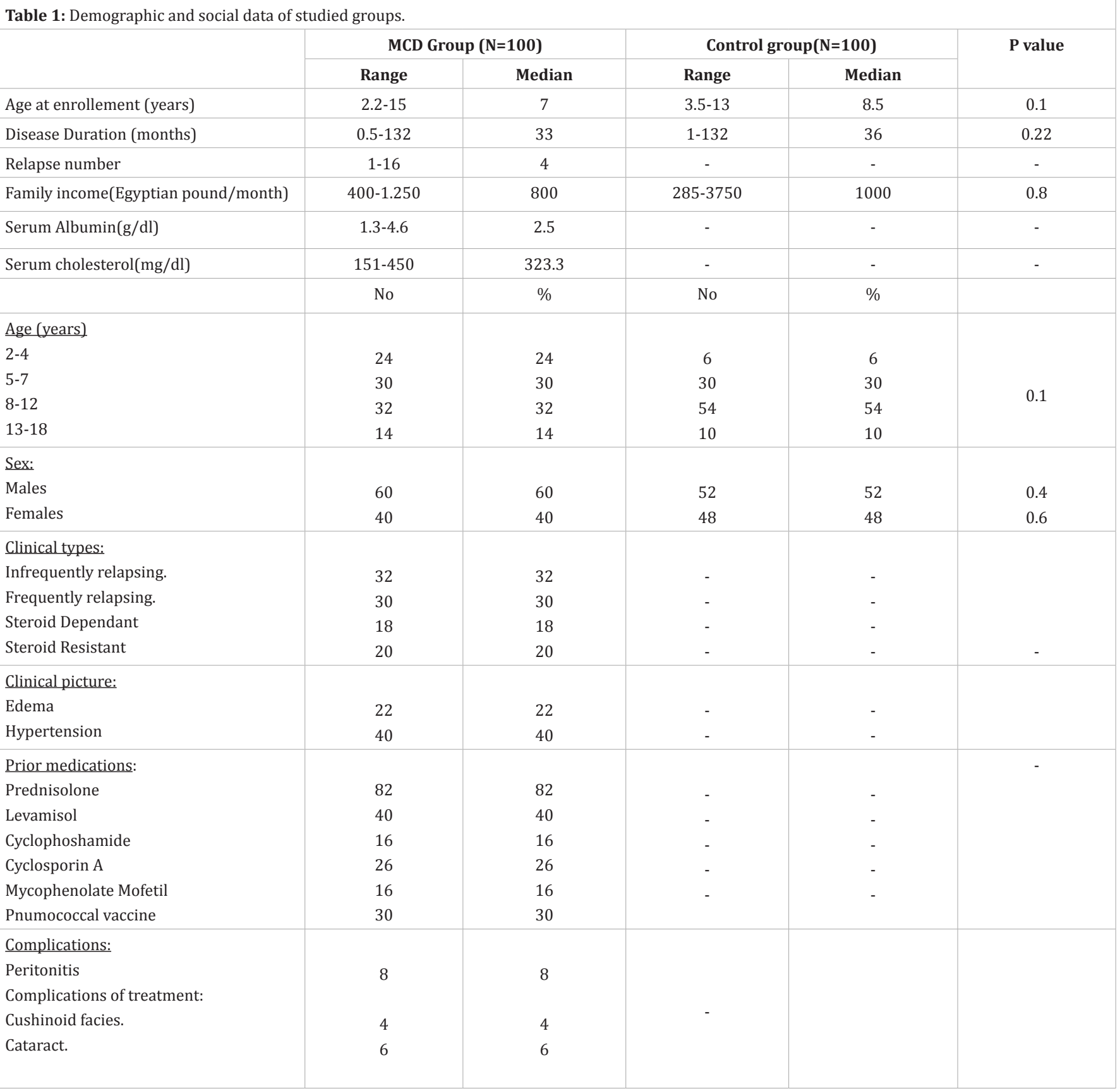


Table 2: PedsQL $\mathrm{L}^{\mathrm{TM}} 4.0$ Generic Core Scale Quality of Life scores in cases and controls.

\begin{tabular}{|l|c|c|c|c|c|}
\hline & \multicolumn{2}{|c|}{ Cases } & \multicolumn{2}{c|}{ Controls } & P value \\
\cline { 2 - 6 } & No (\%) & Range (Median) & No (\%) & Range & (Median) \\
\hline Physical scores: Range (Median) & $100(100 \%)$ & $58.6-75(71.9)$ & $100(100 \%)$ & $58.6-68.8(63)$ & $0.004^{*}$ \\
\hline Emotional scores: Range (Median) & $100(100 \%)$ & $55-65(65)$ & $100(100 \%)$ & $55-65(60)$ & $0.03 *$ \\
\hline Social scores Range (Median) & $100(100 \%)$ & $63.8-75(70)$ & $100(100 \%)$ & $60-70(65)$ & $0.01^{*}$ \\
\hline School scores Range (Median) & $80(80 \%)$ & $56.3-65(63)$ & $98(89 \%)$ & $53-65(65)$ & 0.75 \\
\hline Total score & $80(80 \%)$ & $59-68.8(65)$ & $89(98 \%)$ & $58-65.8(62.2)$ & $0.01 *$ \\
\hline$*$ Pvalue significant $(<0.05)$. & & & & & \\
\hline
\end{tabular}

Table 3: Comparison of PedsQL ${ }^{T M} 4.0$ Generic Core Scale quality of life in different clinical phenotypes of studied MCD

\begin{tabular}{|l|c|c|c|c|}
\hline & $\begin{array}{c}\text { Steroid dependent and } \\
\text { frequently relapsing } \\
\text { (SDNS/FRNS) } \\
\text { (N=48) }\end{array}$ & $\begin{array}{c}\text { Steroid resistant } \\
\text { (SRNS)(N=20) }\end{array}$ & $\begin{array}{c}\text { Infrequently relapsing and } \\
\text { first episode (N=32) }\end{array}$ & P value \\
\hline Physical scores: Range (Median) & $56.3-75(68.8)$ & $65.6-75(71.9)$ & $65.6-75(71.9)$ & 0.72 \\
\hline Emotional scores Range (Median) & $51.3-70(63)$ & $60-71.3(60)$ & $56.3-75(63)$ & 0.3 \\
\hline Social scores Range (Median) & $65-75(70)$ & $63.8-75(73)$ & $60-75(70)$ & 0.66 \\
\hline School scores Range (Median) & $58.8-65(60)$ & $60-65(65)$ & $55-65(60)$ & 0.53 \\
\hline p value is significant <= 0.05 & & & & \\
\hline
\end{tabular}

\section{Discussion}

In pediatric age, patients with chronic diseases as NS, Most of publications and clinical articles focus on the effects of disease activity itself more than long term sequlae. Recently, it is increasingly identified that the impacts on HRQOL is not related to disease activity and therapy but also affected by the cumulative psychological, emotional, behavioural and social effects of the illness as well as drug intake $[3,7]$.

A better understanding of HRQOL in such pediatric patients could help to improve the prognosis of the disease. The Peds $\mathrm{QL}^{\mathrm{TM}}$ 4.0 Generic Core Scale is a brief, standardized generic assessment instrument that evaluate children, adolescents and parents perceptions of HRQOL in this growing age in a systematic way with chronic diseases. The flexibility, reliability, and validity of PedsQL core and modular design made it a simple famous tool in a variety of research publications and clinical applications for chronic health hazards in children and adolescents.

PedsQL ${ }^{\mathrm{TM}}$ 4.0 Generic Core Scale is a previously well used legacy tool that evaluate the body(physical), feelings(emotional), social, and school achivements [8,9].

There were few research studies regarding QOL in children with NS $[3,10]$. Most of published articles have included adult patients or have included patients from Western countries with relatively high socioeconomic standars [3]. So, their occlusions cannot be generalized to other communities. The present study concluded a better QOL in pediatric patients with MCD when compared with other chronic pediatric diseases. This work focused on the commonly missed aspects of care (physical, social, emotional, and school health) in children with
MCD as the most popular variant of INS pediatric age in the context of Egypt as a developing country. More interestingly, we concluded that the assessment of physical, affective, and social performance was better than pediatric patients with other chronic pediatric diseases.This might be attributed to availability of providing our patients different therapeutic modalities of INS free of cost (as we are a governmental University Hospital) including corticosteroid therapy, diuretics, antihypertensives and immunosuppressive chemotherapeutic agents such as cyclosporine A, cyclophosphamide, and mycophenolate mofetil .This led to a good compliance and a good follow-up of included patients. However, an important conclusion was that the school achievement scores in our nephrotic patients were not different from diseased controls in contrast to the other domains in HRQOL.

An analysis of the PedsQL Inventory questionnaire results revealed that all school-going children with INS enrolled in the study, "missed some school days to go to the doctor or hospital" or "missed some school days because of not feeling well."

Even though many of them "did not experience difficulties in class" and "did not have trouble keeping up with school work," the authors believe that the reasons for poor school achievement scores might be partly attributable to frequesnt absence from school secondary to the frequent follow up at their hospitals or clinics for evaluation of the disease activity and assessment of adverse effects related to different medications. The recurrent or relapsing sequlae nature of disease in INS in pediatric age caused irregular school attendance and children may found it was difficult for them to adapt with the burden of missed lessons in addition to interrupted teachers interactions. Moreover, the integration of nephrotic school children into the classroom situation might 
be disturbed secondary to inadequate school environment included lack of health education of teachers who had insufficient knowledge about the nephrotic syndrome and inability to spend adequate compensatory times with these patients [11].

There are few available publications as regard the evaluation of HRQOL in pediatric patients with MCD, especially from developing countries like asian and Arab countries. Rüth et al evaluated QOL, psychological and social adjustment by standardized questionnaire in 45 European pediatric patients with steroid-sensitive NS from the Netherlands. Only the QOL subscale "social function" was impaired while other domains were not affected [3]. In USA, an evaluation of QOL using PedsQL Inventory in 127 American children with prevalent NS as compared to incident NS showed poor scores in social and school performances [2].

In contrast, most Asian studies from India have described the behavioral profile of children with INS, but data regarding QOL in pediatric nephrotic patients was seldom. The emotional and social domains of HRQOL in children with chronic diseases have been assumed to be markedly attributed to ethnic, sociocultural, and socioeconomic status [12]. So the situation different from children in the Western world and could explain the differences in results of our study in comparison to Western countries. This is the first study evaluating HRQOL in acohort of pediatric NS in South India using a validated and flexible measurement model namely PedsQL ${ }^{\text {TM }} 4.0$ Generic Core Scale and is particularly consistent with the paltriness of information in QOL in this patient population. The recruitment of controls with chronic ailments involving other systems provides a reasonable comparative assessment of QOL that has been performed in earlier studies as well [12].

\section{Limitations}

The present study has some limitations. Although factors such as age, gender, duration of illness, clinical type of NS, per capita income, and the immunosuppressive drugs provided for the patients were evaluated for their possible contribution to poor QOL scores, the study could not demonstrate any statistically significant influence. This could be due to a relatively small sample size which was inconsonance. with the short duration of study.

The study is also limited by its cross-sectional nature as a result of which a long-term follow up of this cohort was not undertaken.

One of other limitations of our study is difficult to collect large number of control patients with the same disease so the control group was composed of a variety of chronic clinical conditions; each condition has its own peculiar characteristics that will affect the quality of life of the patient. Moreover, the distribution of these chronic clinical conditions within the control group is also heterogeneous. The heterogeneity, both of the clinical conditions and the distribution of the specific conditions within the control group, provides a bias.

\section{Conclusions}

The present study shows that the overall QOL in children with NS was better when compared with other chronic pediatric illnesses. Clinical phenotypes of NS and demographic parameters did not affect the QOL. Although further studies are needed to confirm the findings of this study and explore the underlying cause of school absences, it is suggested that school level interventions, especially in schools with a larger number of lowincome students should be explored as a strategy to reduce school absenteeism due to NS. Improving attendance in these children requires a multifaceted approach directed toward psychosocial interventions.

\section{Recommendations}

We recommend the health education of parents of nephrotic children through visiting of general pediatricians about knowledge of QOL of their nephrotic children.

Further studies on wider scale should be done for more reliable comparison between QOL of patients with INS and QOL of each separate entity of different chronic diseases which are commonly affecting pediatric age like chronic asthma, epilepsy, chronic hemolytic anemia and others.

\section{References}

1. Gipson DS, Massengill SF, Yao L, Nagaraj S, Smoyer WE, Mahan JD, et al. Management of childhood onset nephritic syndrome. Pediatrics. 2009;124(2):747-757. doi:10.1542/peds.2008-1559

2. Selewski DT, Troost JP, Massengill SF, Gbadegesin RA, Greenbaum LA, Shatat IF, et al. The impact of disease duration on quality of life in children with nephrotic syndrome: A Midwest Pediatric Nephrology Consortium study. Pediatr Nephrol. 2015;30(9):1467-1476. doi:10.1007/s00467-015-3074-x

3. Rüth EM, Landolt MA, Neuhaus TJ, Kemper MJ. Health-related quality of life and psychosocial adjustment in steroid-sensitive nephritic syndrome. J Pediatr. 2004;145(6):778-783. doi:10.1016/j. jpeds.2004.08.022

4. Bagga A, Srivastava RN. Nephrotic syndrome. In: Srivastava RN, Bagga A, 4th edn., Pediatric Nephrology, Jaypee, New Delhi. 2005;159-200.

5. Patro BK, Jeyashree K, Gupta PK. Kuppuswamy's socioeconomic status scale 2010-the need for periodic revision. Indian J Pediatr. 2012;79:395-396. doi:10.1007/s12098-011-0517-7

6. Khothari CR. Research methodology, methods and Techniques,New Age International, In: Khothari CR(ed), 2nd edn., New Delhi, 2012;9597. Isbn(13):978-81-224-2488-1

7. Gipson DS, Selewski DT, Massengill SF, Wickman L, Messer KL, Herreshoff E, et al. Gaining the PROMIS perspective from children with nephrotic syndrome: A Midwest pediatric nephrology consortium study. Health Qual Life Outcomes 2013;11:30. doi:10.1186/14777525-11-30

8. Varni JW, Seid M, Kurtin PS. PedsQL 4.0: Reliability and validity of the Pediatric Quality of Life Inventory version 4.0 generic core scales in healthy and patient populations. Med Care. 2001;39(8):800-812.

9. Varni JW, Burwinkle TM, Seid M, Skarr D. The PedsQL 4.0 as a pediatric population health measure: Feasibility, reliability, and validity. Ambul Pediatr 2003;3(6):329-341. 
10. Shutto Y, Yamabe H, Shimada M, Fujita T, Nakamura N. Quality of life in patients with minimal change nephrotic syndrome. Scientific World Journal 2013;2013:124315. doi:http://dx.doi. org $/ 10.1155 / 2013 / 124315$

11. Guha P, De A, Ghosal M. Behavior profile of children with nephrotic syndrome. Indian J Psychiatry. 2009;51(2):122-126. doi:10.4103/0019-5545.49452
12. Gupta M, Nanda S, Kaushik JS. Quality of life in symptomatic HIV infected children. Indian Pediatr. 2013;50(12):1145-1147. doi:https:// doi.org/10.1007/s13312-013-0300-0 